\title{
Efficacy of Inhaled Steroids in Undiagnosed Subjects at High Risk for COPD*
}

\section{Results of the Detection, Intervention, and Monitoring of COPD and Asthma Program}

\author{
Mieke Albers, MSc; Tjard Schermer, MSc; Guido van den Boom, PhD; \\ Reinier Akkermans, MSc; Constant van Schayck, PhD; \\ Cees van Herwaarden, PhD, MD; and Chris van Weel, PhD, MD
}

\begin{abstract}
Background and aim: COPD leads to a progressive decline of pulmonary function. Family physicians treat a substantial number of patients with COPD and are encouraged to start treatment at as early a stage as is possible. This study analyzed the effectiveness of early inhaled corticosteroid treatment on the decline of pulmonary function in COPD patients.

Patients and setting: Subjects with a rapid decline in lung function (ie, $\mathrm{FEV}_{1}$ decline, $>80 \mathrm{~mL} / \mathrm{yr}$ ) who had never before received a diagnosis of asthma or COPD.

Methods: Two-year, randomized, controlled, double-blind clinical trial of fluticasone propionate (250 $\mathrm{\mu g}$ bid; 24 patients) or placebo ( 25 patients), followed by a 7 -month open-label study in which all subjects received fluticasone propionate. The primary outcome was the post-bronchodilator therapy $\mathrm{FEV}_{1}$, and secondary outcomes were respiratory symptoms, exacerbations, health state, quality of life, and health-care utilization.

Results: After 31 months, there were no statistical differences in post-bronchodilator therapy FEV $_{1}$ between the intervention group and the control group. No statistical differences were observed for symptoms, exacerbations, or quality of life, although tendencies were consistently in favor of treatment. There was no significant impact on the direct or indirect costs.

Conclusions: There are no indications that early treatment with inhaled corticosteroids modifies a rapid decline in lung function or respiratory symptoms and quality of life.
\end{abstract}

(CHEST 2004; 126:1815-1824)

Key words: COPD; early treatment; inhaled corticosteroid; lung function decline

Abbreviations: $\mathrm{BHR}=$ bronchial hyperresponsiveness; $\mathrm{CRQ}=$ chronic respiratory questionnaire; DIMCA $=$ Detection, Intervention, and Monitoring of COPD and Asthma; FP = family physician; MCID = minimal clinically important difference; QALY = quality-adjusted life year

$\mathbf{C}$

OPD is becoming one of the leading causes of disability and death, ${ }^{1}$ and presents an increasing burden of illness to society. In the past, considerable underdiagnosis and undertreatment of chronic airway disease have been reported..$^{2-4}$ At the same time, several studies ${ }^{5-7}$ have reported promising

${ }^{*}$ From the Departments of Family Medicine (Ms. Albers, Mr. Schermer, Dr. van den Boom, Dr. van Weel, and Mr. Akkermans) and Pulmonary Diseases (Dr. van Herwaarden), University Medical Centre, Nijmegen, the Netherlands; and the Department of Family Medicine (Dr. van Schayck), University of Maastricht, the Netherlands.

Dr. van den Boom is currently employed by Novartis Pharma, the Netherlands. Dr. van Schayck received research grants from Boehringer-Ingelheim (2002) and Astra Zeneca (2000). This research has been supported by the Netherlands Organization for Health Research and Development, the Netherlands Asthma Foundation, and GlaxoSmithKline. results with inhaled corticosteroid treatment. In asthma patients, inhaled steroids are effective as first-line therapy. ${ }^{8}$ In COPD patients, the beneficial effects of inhaled steroids remain controversial. ${ }^{9,10}$ The annual rate of lung function decline is greater in patients with COPD and asthma than in healthy subjects. ${ }^{11-13}$ In patients with COPD, inhaled steroid treatment results in the improvement of lung func-

Manuscript received November 11, 2003; revision accepted June 30, 2004.

Reproduction of this article is prohibited without written permission from the American College of Chest Physicians (e-mail: permissions@chestnet.org).

Correspondence to: Mieke Albers, MSc, University Medical Center, Department of Family Medicine [229-HAG], PO Box 9101, 6500 HB Nijmegen, the Netherlands; e-mail: M.Albers@hag. umen.nl 
tion during the first 6 months ${ }^{14-16}$ and some deceleration of lung function decline. ${ }^{17}$ Improvement of health status ${ }^{14,18}$ and reduction of exacerbations ${ }^{14,19}$ also have been reported. These observations have resulted in recommendations to start treatment as early as possible. ${ }^{6}$ For asthma patients, this is included in international guidelines, ${ }^{20}$ but for COPD patients the beneficial effects of early treatment have not yet been demonstrated. From our observation of patients in the practice of family medicine, we hypothesized that the maximum effect of inhaled steroid treatment on lung function decline could be expected if treatment was instituted at the earliest stage of disease, before patients received diagnoses in regular patient care. ${ }^{7}$ This resulted in the establishment of the Dutch Detection, Intervention, and Monitoring of COPD and Asthma (DIMCA) in family practice project in 1991.4,21 The identification of early stages of COPD was later supported by the Global Initiative for Chronic Obstructive Lung Disease classification with the stages 0 (at risk) and I (mild disease). ${ }^{9,10}$ In the current study, we assessed the effects of early inhaled steroid treatment in subjects in whom COPD had not been diagnosed who, prior to intervention, showed a rapid decline of lung function. Subjects receiving early inhaled steroid treatment were compared with subjects in whom this treatment had been postponed for 2 years.

\section{Materials AND Methods}

\section{Design}

Selection for the trial followed a screening and monitoring approach of subjects who had never before received a diagnosis and/or had been treated for asthma or COPD. ${ }^{4}$ All subjects aged 21 to 70 years fulfilling this criterion who were listed in 1 of the 10 family practices involved in the study were invited to the initial screening program (Fig 1). All subjects completed a respiratory symptoms questionnaire, and underwent spirometry and histamine provocation testing. All subjects who reported at least one respiratory symptom and showed airflow obstruction and/or bronchial hyperresponsiveness (BHR) were invited to the second study phase, which consisted of 6 to 24 months of monitoring comprising four to eight lung function measurements. All subjects with an annual pre-bronchodilator therapy $\mathrm{FEV}_{1}$ decline of $\geq 80 \mathrm{~mL}$ were considered to be at risk for developing COPD and were invited into the current study (Fig 1).

To study the effects of early inhaled steroid intervention, a 2-year, randomized, double-blind, placebo-controlled trial was initiated (also referred to as early inhaled steroid treatment), followed by an open-label extension period of 7 months (also referred to as postponed inhaled steroid treatment). The allocation of subjects to the intervention and control groups followed a block randomization within each family practice. Study medication (fluticasone propionate, $250 \mu \mathrm{g}$ bid administered by metered-dose inhaler, and matching placebo) was prepared by the manufacturer (GlaxoSmithKline; Research Triangle Park, NC). Fluticasone and placebo were packed in identical canisters.
The patients' family physician (FP) handed out a new set of study medication every 3 months. To record drug compliance, the old canisters were taken in and weighed. Subjects also recorded their medication use in a trial diary. Individual compliance rates were determined by expressing the number of puffs consumed ( $i e$, the dosages provided minus dosages returned) as a percentage of the amount prescribed during the study. ${ }^{22}$ When noncompliance was observed during trial visits, subjects were reminded of the need to adhere to prescribed medication and were instructed in proper use. Subjects were permitted to use short-acting bronchodilator agents as rescue medication. In case of an exacerbation, a 10-day course of therapy with oral prednisolone was advised.

Post-bronchodilator therapy $\mathrm{FEV}_{1}$ decline served as the primary outcome. During the monitoring phase preceding the trial, the observed mean annual $\mathrm{FEV}_{1}$ decline for the subjects who were eligible for study participation was $>200 \mathrm{~mL} / \mathrm{yr}$. Halving this rate to $100 \mathrm{~mL} / \mathrm{yr}$ was considered a relevant treatment effect. A per-protocol participation of 56 subjects (28 per group) was required to detect this difference (assumptions: $1-\beta=0.80$; $\alpha=0.05 ; \mathrm{SD}=132 \mathrm{~mL}$ ). Secondary outcomes were respiratory symptoms, exacerbations, health state, quality of life, and healthcare utilization. The medical ethics review board of the University Medical Centre Nijmegen approved the study. Subjects gave their written informed consent.

\section{Measurements}

Lung Function, Reversibility, and BHR: Lung function measurements were performed in a lung function laboratory (Microspiro HI-298 spirometer; Chest Corporation; Tokyo, Japan) ${ }^{24}$ following American Thoracic Society standards. ${ }^{23}$ Measurements were scheduled at baseline and repeated every 6 months. The European Community for Coal and Steel predicted values were calculated. ${ }^{25}$ Reversibility was defined as a $10 \%$ change in the $\mathrm{FEV}_{1}$ percentage of predicted value 15 min after the inhalation of $800 \mu \mathrm{g}$ salbutamol by spacer. A histamine provocation test ${ }^{26}$ was performed to assess bronchial responsiveness. Subjects were considered to have experienced BHR if the provocative dose of histamine causing a $20 \%$ drop in $\mathrm{FEV}_{1}$ was $<8 \mathrm{mg} / \mathrm{mL}$.

Respiratory Symptoms and Exacerbations: Subjects recorded the presence and severity of four respiratory symptoms (ie, cough, shortness of breath, wheeze, and productive cough) on diary cards. A weekly symptom score was calculated based on the number of reported symptoms in the past week (minimum, 0 ; maximum, 4). A week with a symptom score of 0 was defined as a symptom-free week. An exacerbation was defined as an acute episode that required medical attention during which at least two of the following three criteria had to be fulfilled: increased (productive) cough, and/or dyspnea, and/or wheezing; change in sputum color; increased use of bronchodilators. ${ }^{7}$

Quality of Life and Health State: Disease-specific quality of life was measured at baseline and every 6 months using the interviewer-administered version of the chronic respiratory questionnaire (CRQ). ${ }^{29} \mathrm{~A}$ within-subject improvement of $0.5 \mathrm{U}$ at the end of the 2-year observation period was considered to be a minimal clinically important difference (MCID). ${ }^{30}$ Preference-based utilities were measured every 6 months by standard gamble procedure using the Maastricht utility measurement questionnaire. ${ }^{27}$ Quality-adjusted life years (QALYs) were determined by calculating the area under the utility-time curve for each subject. One additional QALY is interpreted as one additional year in perfect health for one subject.

\section{Health-Care Resource Use and Productivity Losses}

Consultations and prescriptions for respiratory medication were recorded by the patients' FPs and were verified by the 


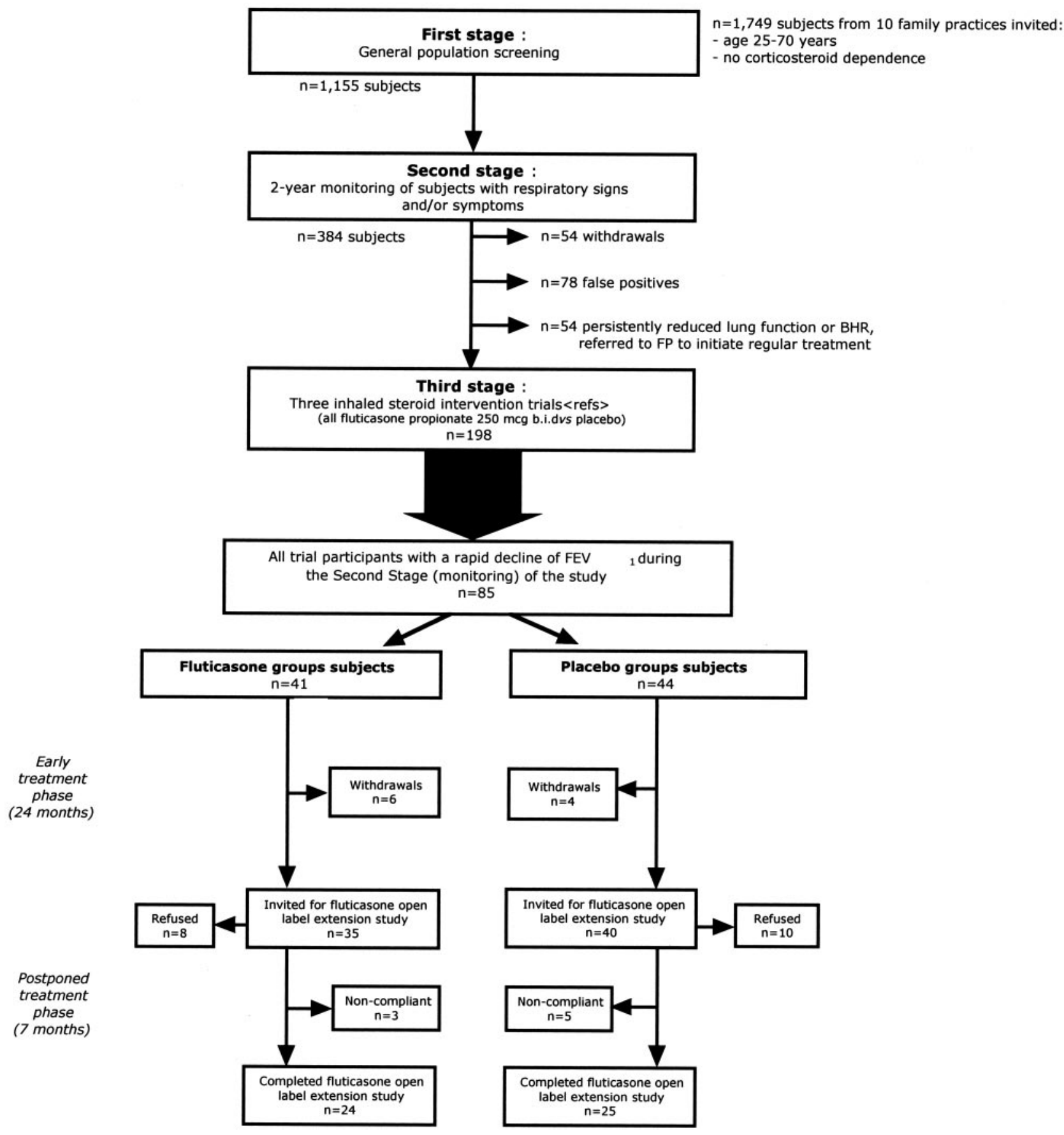

FIGURE 1. Flow chart of the initial general population cohort in the DIMCA program, and the trial population derived from it. This article reports on the subjects having a rapid decline of $\mathrm{FEV}_{1}$ from the original trials who were included in a per-protocol analysis.

investigators through medical record audit and a standardized interview that was conducted every 3 months with study subjects. Medication cost included pharmacy cost and the value added tax. Subjects recorded their use of over-the-counter medications and the number of days they had been unable to perform paid or unpaid work in the diary. Indirect costs were assessed using the Human Capital Approach. Dutch guilders were converted to Euros $(€)$. One day of lost work was valued as $8 \mathrm{~h}$ times $€ 8.18$ per hour. For conversion to US dollars, Euros should be multiplied by a factor 0.912 , based on the 2000 Purchasing Power Parities, as issued by the Organization for Economic Cooperation and Development.

\section{Statistical Analysis}

Subjects who participated in the 2-year early intervention trial and in the 7-month open-label extension with fluticasone treatment, and who had used at least $70 \%$ of their trial medication 
were included in a per-protocol analysis. Treatment groups were compared on baseline characteristics using appropriate univariate statistical tests. Because of the observed initial increase of the post-bronchodilator therapy $\mathrm{FEV}_{1}$, a linear regression model with one breaking point showed the best fit for the course of this parameter over time. This so-called piecewise model was used to analyze both early and postponed treatment effects. For early treatment, the model discriminated between the initiation of treatment ( $i e, 0$ to 3 months) and ongoing treatment (ie, 4 to 24 months), and for postponed treatment it discriminated between the initiation of treatment (ie, 24 to 26 months) and ongoing treatment (ie, 27 to 31 months). The mean change in postbronchodilator therapy $\mathrm{FEV}_{1}$ over time was estimated by a mixed linear effect model for repeated measurements, ${ }^{31}$ which included baseline $\mathrm{FEV}_{1}$, age, gender, height, and smoking history as covariates. The paired $t$ test was used to compare the change per period. Differences in symptom-free weeks and CRQ scores were tested using $t$ test statistics. The course of the CRQ total and domain scores was analyzed using a mixed-effects model. Poisson analysis was used to analyze the number of exacerbations. Utilities were converted to QALYs by calculating the area under the curve for the periods from 0 to 24 months and from 24 to 31 months. The mean differences in QALYs were tested using the $t$ test, and longitudinal analysis (a mixed-effects model with correction for baseline values) was used to estimate utility over time. A statistical software package (SAS, version 6.12; SAS Institute; Cary, NC) was used for all analyses.

\section{Results}

During the DIMCA monitoring phase, 85 subjects with a rapid decline in lung function were identified (Fig 1). Forty-one subjects were randomized to receive early fluticasone treatment, and 44 subjects were randomized to receive placebo treatment. Of these subjects, $57(67 \%)$ participated in the openlabel, postponed fluticasone study. The per protocol analysis included all 49 subjects who finished both the early treatment trial and the open-label extension study, and were $\geq 70 \%$ compliant on trial medication use. The withdrawal, refusal, and noncompliance of subjects did not show any selection bias (results not presented). For the early fluticasone treatment group (24 patients), the mean annual decline prior to treatment was $210 \mathrm{~mL}$ (SD, 130 $\mathrm{mL}$ ), and for the placebo group (25 patients) the mean annual decline was $224 \mathrm{~mL}(\mathrm{SD}, 134 \mathrm{~mL})$. At the start of the trial, the treatment groups were not different in terms of age, post-bronchodilator therapy $\mathrm{FEV}_{1}, \mathrm{BHR}$, or reversibility (Table 1 ), but the early treatment group included more ever-smokers and had a history of more pack-years of smoking.

\section{Clinical Effects}

Lung Function: There were no statistically significant differences in the course of the post-bronchodilator therapy $\mathrm{FEV}_{1}$ over time between the treatment groups (Fig 2). The estimated mean changes of the post-bronchodilator therapy $\mathrm{FEV}_{1}$ during the
Table 1-Baseline Characteristics of Study Subjects by Treatment Group*

\begin{tabular}{|c|c|c|}
\hline \multirow[b]{2}{*}{ Characteristics } & \multicolumn{2}{|c|}{ Treatment Groups } \\
\hline & $\begin{array}{l}\text { Fluticasone Propionate } \\
\qquad(\mathrm{n}=24)\end{array}$ & $\begin{array}{l}\text { Placebo } \\
(\mathrm{n}=25)\end{array}$ \\
\hline Age, yr & $51.8(10.3)$ & $47.9(10.0)$ \\
\hline Female gender, \% & 58.3 & 40.0 \\
\hline Height, cm & $168(10)$ & $172(9)$ \\
\hline \multicolumn{3}{|l|}{ Smoking history at entry } \\
\hline$\%$ ever & 75.0 & 48.0 \\
\hline Pack-years $\uparrow$ & $9(0.5-24)$ & $0(0-8)$ \\
\hline Allergy, \% & 54.5 & 45.8 \\
\hline $\begin{array}{l}\text { No bronchial symptoms } \\
\text { reported, \% }\end{array}$ & 37.5 & 52.0 \\
\hline \multicolumn{3}{|l|}{ Pre-bronchodilator therapy } \\
\hline $\mathrm{L}$ & $2.62(0.75)$ & $3.02(0.66)$ \\
\hline$\%$ predicted & $88.3(14.5)$ & $91.2(14.4)$ \\
\hline \multicolumn{3}{|l|}{$\begin{array}{l}\text { Post-bronchodilator } \\
\text { therapy } \mathrm{FEV}_{1}\end{array}$} \\
\hline $\mathrm{L}$ & $2.73(0.75)$ & $3.10(0.72)$ \\
\hline$\%$ predicted & $91.9(12.0)$ & $94.0(14.8)$ \\
\hline $\begin{array}{l}\text { Post-bronchodilator } \\
\text { therapy } \mathrm{FEV}_{1} / \mathrm{IVC}\end{array}$ & $0.75(0.06)$ & $0.75(0.07)$ \\
\hline $\begin{array}{l}\text { Reversibility of } \mathrm{FEV}_{1}, \% \\
\text { predicted }\end{array}$ & 2.93 & 1.62 \\
\hline $\mathrm{PC}_{20}$ histamine, $\mathrm{mg} / \mathrm{mL}+$ & 3.20 & 3.62 \\
\hline $\mathrm{BHR}, \%$ & 66.7 & 52.0 \\
\hline CRQ score§ & $5.9(0.86)$ & $6.1(0.79)$ \\
\hline Dyspnea domain & $6.2(1.2)$ & $6.5(1.2)$ \\
\hline Emotions domain & $5.6(0.9)$ & $5.7(0.9)$ \\
\hline Fatigue domain & $5.5(1.1)$ & $5.7(1.1)$ \\
\hline Mastery domain & $6.3(0.8)$ & $6.6(0.6)$ \\
\hline \multicolumn{3}{|l|}{ Utility\| } \\
\hline Reference health state & $0.55(0.16)$ & $0.61(0.21)$ \\
\hline Own health state & $0.79(0.17)$ & $0.85(0.13)$ \\
\hline $\begin{array}{l}\text { *Values given as mea } \\
\mathrm{PC}_{20}=\text { concentration of } \\
\geq 20 \% \text {. } \\
\uparrow \text { Values given as median }( \\
+ \text { Values given as geometri } \\
\$ \text { Range, } 1 \text { to } 7 \text {. } \\
\| \text { Range, } 0 \text { to } 1 .\end{array}$ & $\begin{array}{l}\text { (SD), unless other } \\
\text { histamine provoking a } \\
\text { nterquartile range). } \\
\text { mean. }\end{array}$ & $\begin{array}{l}\text { wise indicated. } \\
\text { fall in } \mathrm{FEV}_{1} \text { of }\end{array}$ \\
\hline
\end{tabular}

subsequent study phases are shown in Table 2. For other pulmonary function measures (ie, mean maximal expiratory flow and inspiratory vital capacity), similar patterns were found (data not shown). An explorative subgroup analysis on smoking behavior, allergy, and $\mathrm{BHR}$, in addition to the rapid $\mathrm{FEV}_{1}$ decline, did not modify the effect of those using fluticasone relative to those using placebo (data not shown).

Respiratory Symptoms and Exacerbations: During the monitoring phase preceding the trial, eight exacerbations were recorded in the group that was later allocated to receive fluticasone treatment, and three exacerbations occurred in the group that was later allocated to receive placebo treatment. During the 


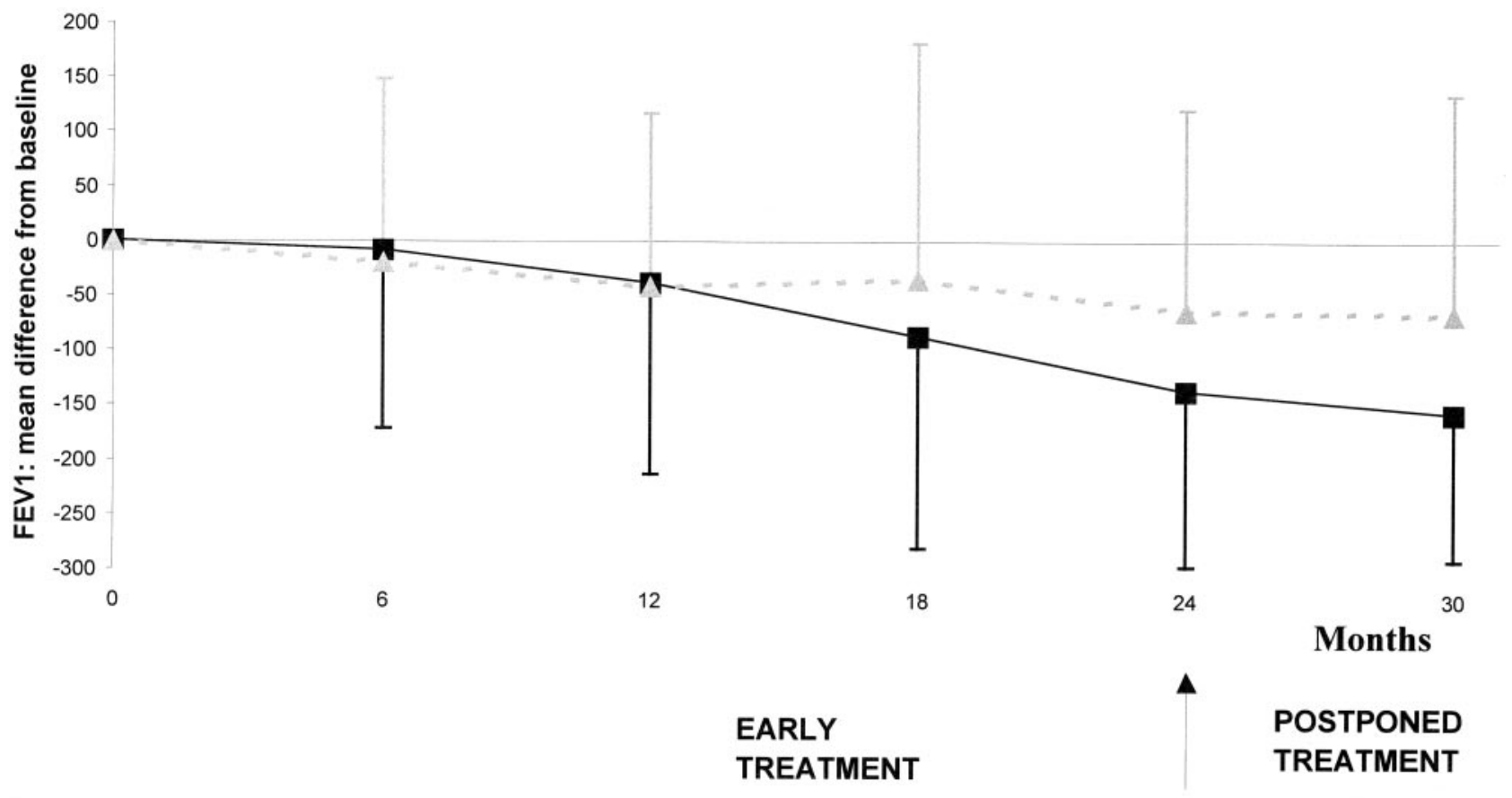

- Early Fluticasone Propionate treatment

Placebo respectively postponed Fluticasone Propionate treatment

FIGURE 2. Course of the mean post-bronchodilator therapy $\mathrm{FEV}_{1}$ during treatment. Bars indicate SEs.

early treatment trial, three exacerbations were recorded in the fluticasone group vs seven exacerbations in the placebo group. During the open-label extension period, one additional exacerbation was recorded in the group that continued to receive fluticasone, and three exacerbations in the postponed fluticasone treatment group. Poisson analysis did not show a difference in exacerbations between the groups for the subsequent periods $(\mathrm{p}=0.18)$. Of the 25 exacerbations reported, $17(68 \%)$ were first exacerbations.

During early treatment, the number of symptomfree weeks did not differ between the early treat- ment and postponed treatment groups $(61.5 \%$ vs $71.5 \%$, respectively; $\mathrm{p}=0.34$ ). During postponed treatment, these percentages were somewhat different (early treatment group, 56.5\% symptom-free weeks; postponed treatment group, $76.5 \%$ symptomfree weeks; $\mathrm{p}=0.06)$.

Quality of Life and Health State: Baseline CRQ scores indicated a mild impairment in quality of life (Table 1). During early treatment, there was no difference between the treatment groups in either the CRQ total score or the domain score (Fig 3). The

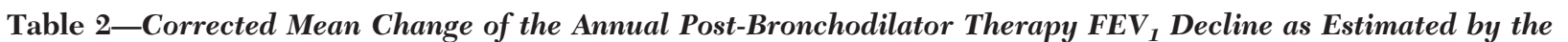
(Piecewise) Regression Models*

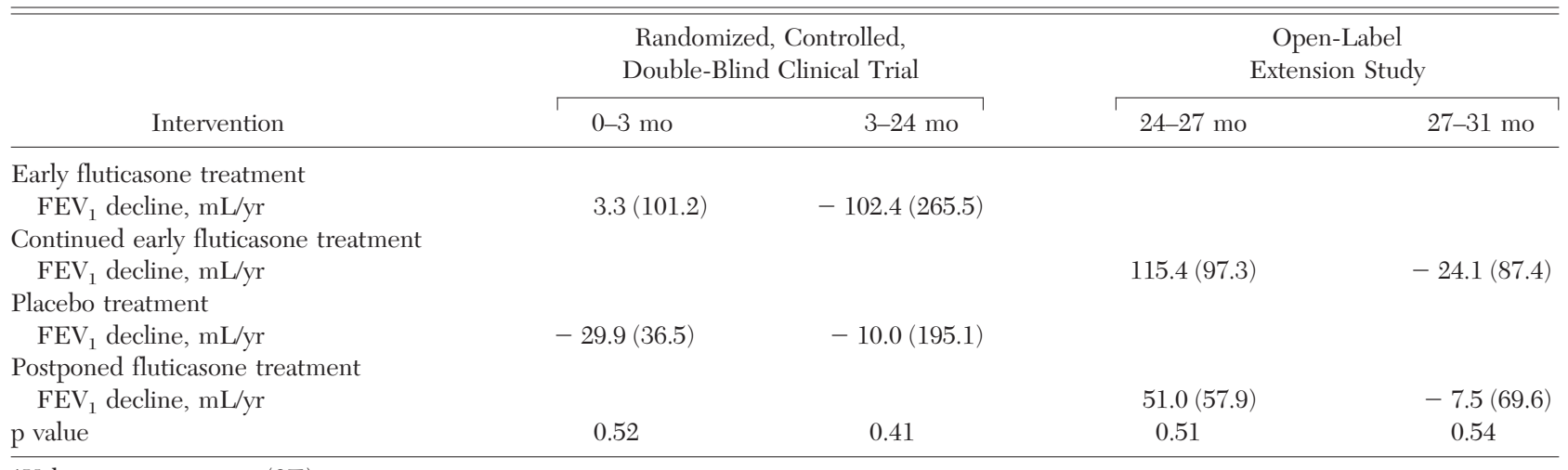

*Values given as mean (SE). 


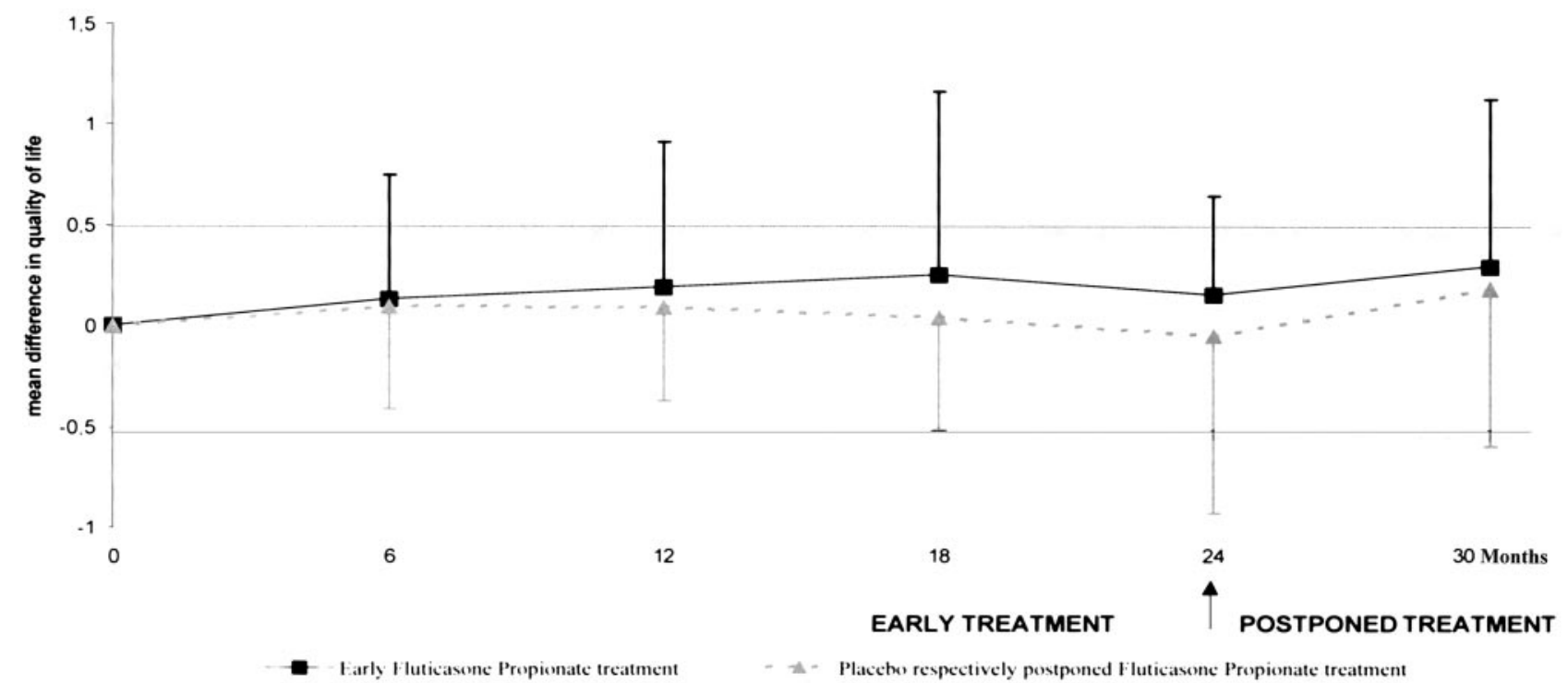

FiguRE 3. Disease-specific, health-related quality of life (the CRQ). Bars indicate SEs.

proportion of subjects with an MCID on the CRQ was $21 \%$ in the early treatment group vs $24 \%$ in the placebo group $(\mathrm{p}=0.79)$. The dyspnea score $(25 \%$ vs $12 \%$, respectively), the fatigue score ( $21 \%$ vs $16 \%$, respectively), and the mastery domain scores ( $17 \%$ vs $8 \%$, respectively) were consistently higher in the fluticasone treatment group than in the placebo treatment group (not statistically significant). For the CRQ emotions domain, the proportion of subjects was higher in the placebo group than in the fluticasone treatment group (25\% vs $32 \%$, respectively; not statistically significant). After postponed treatment, the proportions of subjects with an MCID in quality of life were $14 \%$ for the postponed treatment group and $33 \%$ for the early treatment group $(p=0.12)$.

There were no significant differences in baseline utilities between the subjects' own health state and the hypothetical health state (Table 1). During both the early and postponed treatment, a large proportion (ie, 36 to $45 \%$ ) of the health state descriptions collected did not indicate any impairment of generic quality of life. The course of the mean utility of the subject's health status is shown in Figure 4. Early treatment resulted in a mean difference of 0.0244 in

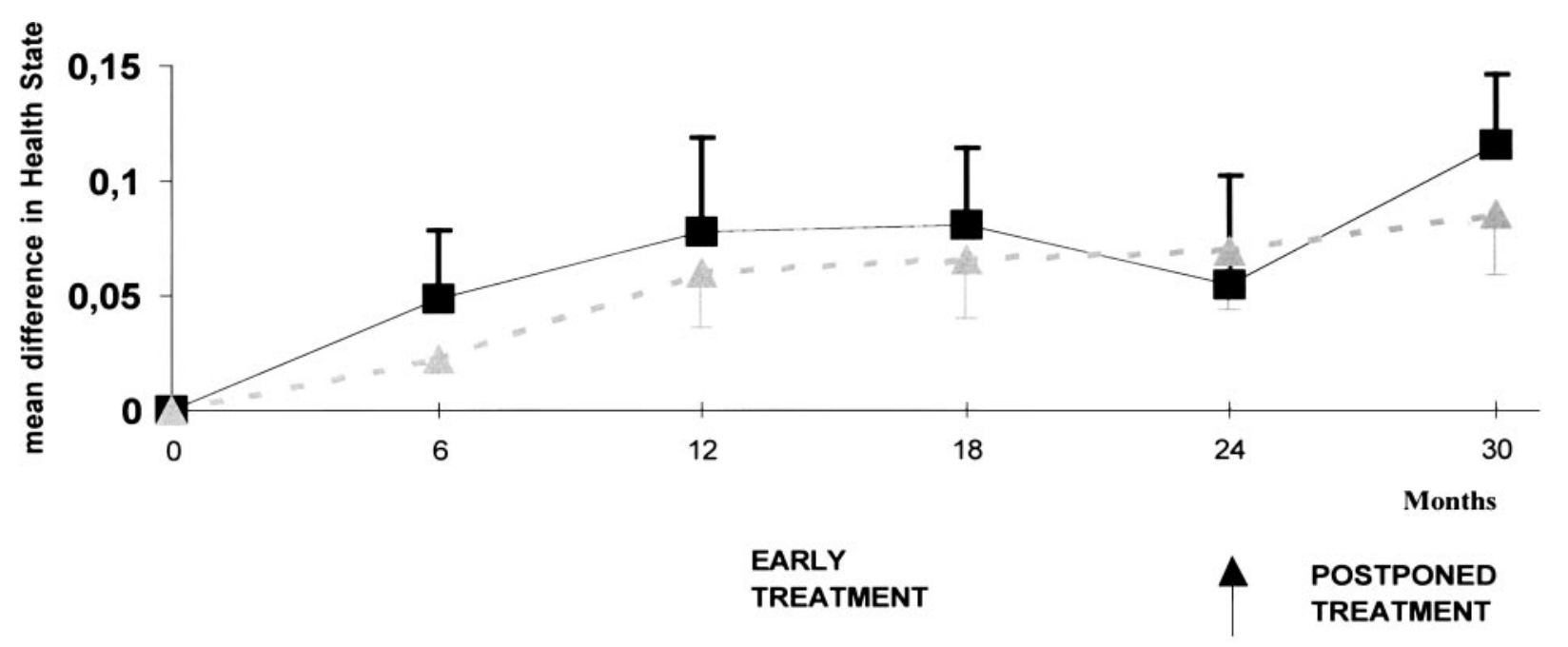

- Early Fluticasone Propionate treatment

placebo respectively postponed Fluticasone Propionate treatment

Figure 4. Preference-based valuations of the subjects' own health status (standard gamble utilities).

Bars indicate SEs. 
favor of fluticasone ( $\mathrm{p}=0.72$; Table 3 ). Expressed in QALYs, health state was indicative of the effect of treatment. For early treatment, 1.22 QALYs were gained per year per 100 treated subjects (95\% confidence interval, -5.5 to 7.9 ). Postponed treatment resulted in a mean difference of 0.0038 in favor of fluticasone treatment $(p=0.87$; Table 3$)$. Expressed in QALY gains, this amounts to 0.76 QALYs per year per 100 treated subjects $(95 \%$ confidence interval, -7.0 to 8.6$)$. In the early treatment period, the estimated mean difference in utility was 0.0099 per year $(p=0.75)$. Assuming that this effect persists in subsequent years, 0.99 QALYs would be gained by early fluticasone intervention per 100 treated subjects per year.

Health-Care Resource Use: During early treatment, the mean annual cost of experimental medication was € 310 per subject (Table 4). The costs of nonexperimental respiratory medication in the fluticasone group and the placebo group were $€ 10.99$ and $€ 17.39$, respectively $(\mathrm{p}=0.34$ ). The average number of prescriptions written per year for respiratory medication was lower in the fluticasone treatment group ( 0.70 prescriptions per subject) compared to that in the placebo group (1.19 prescriptions per subject). The total mean health-care cost was similar in both groups $(p=0.87)$. There were no referrals to chest physicians or respiratory disease-related hospital admissions. The cost of fluticasone treatment constituted the major part of the direct health-care cost. The average number of days that a subject had been unable to perform his or her normal activities due to respiratory symptoms was 3.1 days in the fluticasone group and 2.5 days in the placebo group. The associated mean indirect cost was €203.05 and €164.52, respectively $(p=0.65)$. During postponed fluticasone treatment, the cost of nonexperimental respiratory medication remained higher (€8.03 vs €15.80, respectively; $\mathrm{p}=0.15$ ). The use of nonexperimental respiratory medication during the trial (placebo treatment) appeared to be highly correlated with the extent of use during the extension period (postponed fluticasone treatment; $r=0.90$ ). During postponed fluticasone

Table 3-The (Piecewise) Calculated Gain in Health State

\begin{tabular}{ccccc}
\hline \hline & \multicolumn{2}{c}{ Early Treatment } & \multicolumn{2}{c}{ Postponed Treatment } \\
\cline { 2 - 3 } Variable & $\begin{array}{c}\text { Placebo } \\
(\mathrm{n}=25)\end{array}$ & $\begin{array}{c}\text { Fluticasone } \\
(\mathrm{n}=24)\end{array}$ & $\begin{array}{c}\text { Postponed } \\
\text { Fluticasone } \\
(\mathrm{n}=23)\end{array}$ & $\begin{array}{c}\text { Early } \\
\text { Fluticasone } \\
(\mathrm{n}=23)\end{array}$ \\
\hline QALY gains & 0.0890 & $0.1134^{*}$ & 0.0457 & $0.0419 \uparrow$ \\
\hline
\end{tabular}

$* \mathrm{p}=0.72$.

$\mathrm{t}_{\mathrm{p}}=0.87$. treatment, the average number of prescriptions per year decreased, mainly in the early fluticasone treatment group. The average cost of health-care resource use remained constant $(p=0.93)$, and there were no referrals or hospital admissions. Loss of productivity changed over time. In the early fluticasone treatment group, the number of days lost showed a threefold increase $(p=0.05)$, whereas in the postponed treatment group the increase was twofold $(p=0.14)$. The associated indirect costs were €589 and €334, respectively. There was no significant difference in indirect costs between the groups $(\mathrm{p}=0.27)$.

\section{Discussion}

In the DIMCA program, subjects in whom COPD formerly had been undiagnosed but had objective signs of COPD had their condition detected and treated in a family practice. Although chronic airway obstruction can be identified in such an early stage, a final diagnosis, differentiating between asthma and COPD, often is not yet possible. As subjects with airway obstruction who show a rapid decline in lung function bear an increased risk for the development of COPD, this study focused on this particular group. Following active detection, subjects were treated at an earlier stage than would have been possible in a regular general practice setting. In the current study, the effect of treatment was measured by the change in the course of post-bronchodilator therapy $\mathrm{FEV}_{1}$. In conformance with our initial hypothesis, lung function follow-up values were analyzed in a model that allows for differentiation between the initial improvement of lung function and the long-term mediation of the course of lung function decline, in subjects receiving both early and postponed treatment. However, there was no initial improvement among the fluticasone-treated subjects in the group of rapid decliners. Also, the hypothesis that a delay in treatment would cause irreversible damage could not be confirmed. The decline in lung function in the early intervention group did not differ from that after postponed intervention. A post hoc power analysis based on the actual number of trial participants (49 participants, instead of the assumed 56 participants) revealed that with the per-protocol participation of 49 subjects a treatment difference of $105 \mathrm{~mL} / \mathrm{yr}$ (80\% power; $\alpha=0.05$; SD, $132 \mathrm{~mL} / \mathrm{yr}$ ) could have been detected.

Several early studies ${ }^{5,6}$ showing promising results with inhaled steroid treatment expected maximum benefit (ie, the prevention of the irreversible loss of lung function) if treatment was instituted as early as possible in the development of the disease. In a metaanalysis, ${ }^{32}$ it was observed that patients with 
Table 4-Average Cost (in Euros) for Early and Postponed Fluticasone Propionate Treatment*

\begin{tabular}{|c|c|c|c|c|c|c|c|c|}
\hline \multirow[b]{3}{*}{ Costs } & \multicolumn{4}{|c|}{$0-24 \mathrm{mo}$} & \multicolumn{4}{|c|}{ 24-31 mo } \\
\hline & \multicolumn{2}{|c|}{$\begin{array}{l}\text { Placebo Treatment } \\
\qquad(\mathrm{n}=25)\end{array}$} & \multicolumn{2}{|c|}{$\begin{array}{c}\text { Early Fluticasone } \\
\text { Treatment }(\mathrm{n}=24)\end{array}$} & \multicolumn{2}{|c|}{$\begin{array}{l}\text { Postponed Fluticasone } \\
\text { Treatment }(\mathrm{n}=25)\end{array}$} & \multicolumn{2}{|c|}{$\begin{array}{c}\text { Continued Fluticasone } \\
\text { Treatment }(\mathrm{n}=24)\end{array}$} \\
\hline & Units & Cost & Units & Cost & Units & Cost & Units & Cost \\
\hline \multicolumn{9}{|l|}{ Direct medical cost } \\
\hline Fluticasone propionate (trial medication) $\uparrow$ & & 0.00 & 6.09 & 310.18 & 6.09 & 310.18 & 6.09 & 310.18 \\
\hline Bronchodilators幸 & 0.61 & 9.30 & 0.18 & 3.27 & 0.46 & 7.00 & 0.14 & 1.45 \\
\hline Antibiotics幸 & 0.26 & 3.54 & 0.20 & 2.48 & 0.13 & 2.64 & 0.14 & 3.55 \\
\hline Corticosteroids (noninhaled) $\stackrel{+}{+}$ & 0.07 & 0.46 & 0.22 & 2.62 & 0.14 & 0.85 & 0.07 & 0.94 \\
\hline Other prescribed respiratory medication $+\underset{+}{+}$ & 0.26 & 3.03 & 0.09 & 0.57 & 0.20 & 1.84 & 0.00 & 0.00 \\
\hline OTC medication & & 1.04 & & 2.04 & & 3.10 & & 2.09 \\
\hline FP consultation & 0.34 & 5.96 & 0.32 & 5.35 & 0.33 & 5.52 & 0.31 & 5.36 \\
\hline Specialist consultation & & 0.00 & & 0.00 & & 0.00 & & 0.00 \\
\hline \multicolumn{9}{|l|}{ Indirect cost } \\
\hline Days lost from work (unpaid) & 2.51 & 164.52 & 3.10 & 203.05 & 5.09 & 333.59 & 9.00 & 588.98 \\
\hline \multicolumn{9}{|l|}{ Total cost } \\
\hline Fluticasone (trial medication) $\uparrow$ & & 0.00 & 6.09 & 310.18 & 6.09 & 310.18 & 6.09 & 310.18 \\
\hline All respiratory medication & 1.19 & 17.39 & 0.70 & 10.99 & 0.94 & 15.80 & 0.36 & 8.03 \\
\hline Direct medical & & 23.35 & & 326.53 & & 331.51 & & 323.38 \\
\hline Indirect & 2.51 & 164.52 & 3.10 & 203.05 & 5.09 & 333.59 & 9.00 & 588.98 \\
\hline Direct + indirect & & 187.87 & & 529.58 & & 665.11 & & 912.36 \\
\hline
\end{tabular}

*Costs are standardized to 1 year. OTC $=$ over the counter.

† Based on daily doses cost.

+ Prescriptions.

clearly defined moderate-to-severe COPD had a slowdown in the course of lung function decline during 2 years of treatment with a relatively high dosage of inhaled corticosteroids. A number of large randomized clinical trials ${ }^{13,14,33,34}$ evaluating the efficacy of inhaled steroids have demonstrated initial improvement in lung function. However, these studies $^{13,14,33,34}$ confirmed the findings of our study that inhaled steroid treatment does not substantially modify the course of pulmonary function.

Although randomized at the start, the treatment groups did not have a similar baseline health profile. Subjects who were later allocated to early fluticasone treatment had a slightly lower lung function and showed less reversibility. Although not statistically significant, they were found to be older, shorter, more often allergic, less often without symptoms, and more (often) to be smokers. This might indicate that the early treatment group had a somewhat worse prognosis at baseline. This is also illustrated by the change in lung function (Table 1). Rapid decline in lung function may be considered a strong indicator of COPD; however, its (short-term) value in evaluating treatment can be debated. Judged by the percentage change in the two groups, however, the actual gains in health in the two groups were quite comparable.

At the time of planning this study (1992), change in lung function was the predominant concept of the outcome of COPD,, 17 and for that reason it was chosen as the primary outcome measure. Nowadays, it is recognized that a rapid decline is not the only clinical characteristic of early stage COPD. ${ }^{10}$

The rationale for the early treatment of COPD is its progressive nature and poor response to therapy once the disease is fully developed. But its early diagnosis is problematic, as the initial minimal symptoms and pulmonary function impairment are difficult to distinguish from self-limiting respiratory illness or asthma. For that reason, participants were included in this study after an observed decline in $\mathrm{FEV}_{1}$ of $>80 \mathrm{~mL} / \mathrm{yr}$ over 2 years, which is an observation period during which other studies had been able to find clinically relevant developments in pulmonary function. ${ }^{32}$ Irreversible loss of pulmonary function is characteristic of COPD, although (partial) irreversibility is also seen in asthma. Some patients in this study had BHR and were allergic, which are features of asthma. However, BHR is also often present in COPD patients. ${ }^{35}$

Today, the emphasis is less on pulmonary function development as the outcome of the treatment of COPD, but is more on symptoms, exacerbations, health effects, and cost. However, in an exploratory analysis these secondary effect measures did not show a relevant effect of early fluticasone treatment. There was no significant increase in QALYs, nor were there any differences in exacerbation rate, health-related quality of life, or respiratory symptoms. Considering the economic consequences, 
there was no difference in total mean health-care cost or indirect cost. Incremental costs were predominantly due to the use of experimental medications. As subjects for this study were recruited in the very early stage of their disease (before they had received a diagnosis from a regular care physician), room for improvement is likely to be small in any disease-related outcome measure. However, several tendencies on fluticasone treatment effects were identified. Preceding intervention, more exacerbations were recorded in the group that was later allocated to fluticasone treatment. During fluticasone treatment, improvement was found in the fluticasone group, suggesting a protective effect of therapy with inhaled steroids on the exacerbation rate. Furthermore, subjects in the postponed treatment group reported more symptom-free weeks after the initiation of fluticasone treatment. At baseline, subjects rated their health as relatively good. In both groups, there was a slight improvement in health status, implying an in-care effect. Early treatment did not show significant improvement in the subjects' health state, but the results were suggestive for some health benefit. Furthermore, it was remarkable that the difference in the use of nonexperimental respiratory medication was mainly due to bronchodilator use. In the placebo group, medication use remained constant throughout the open-label extension period. This may reflect a person's adaptation to medication use. Between groups, the main difference in health-care utilization had to be attributed to days lost from work, either paid or unpaid. After 2 years, the average number of days lost from work increased in both groups, but mostly in the group with early treatment. As these data do not allow for a more advanced analysis, it remains unclear whether subjects' health became worse despite treatment or whether group prognoses were different at the start.

The DIMCA project is unique in that it has set out to study subjects who were in the state of developing COPD before signs and symptoms had brought them to seek regular medical care. In this phase, no effect from inhaled steroid treatment on lung function could be found. This implies that for this group the delay in initiating treatment did not result in the irreversible loss of lung function. On secondary outcomes, the results of both early and postponed treatment were suggestive of subtle health gains in respiratory symptoms, exacerbation rate, and health state. FPs therefore should continue to focus on the early detection and treatment of those visiting them who have respiratory signs and symptoms. But the study did not present evidence of the benefits of screening in order to begin treatment in the preclinical phase of the disease.
ACKNOWLEDGMENT: The authors thank Lea Peters for her indispensable research assistance, and Joke Grootens for her assistance in compiling the cost data.

\section{REFERENCES}

1 Murray CJL, Lopez AD. Alternative visions of the future: projecting mortality and disability, 1990-2020. In: Murray CJL, Lopez AD, eds. The global burden of disease. Cambridge, MA: The Harvard School of Public Health, on behalf of the World Health Organization and the World Bank, 1996; 361-395

2 Tirimanna PR, Van Schayck CP, Den Otter JJ, et al. Prevalence of asthma and COPD in general practice in 1992: has it changed since 1977? Br J Gen Pract 1996; 46:277-281

3 Stang P, Lydick E, Silberman C, et al. The prevalence of COPD: using smoking rates to estimate disease frequency in the general population. Chest 2000; 117(suppl):354s-359s

4 Van den Boom G, Van Schayck CP, Rutten-van Molken MP, et al. Active detection of chronic obstructive pulmonary disease and asthma in the general population: results and economic consequences of the DIMCA program. Am J Respir Crit Care Med 1998; 158:1730-1738

5 Dompeling E, Van schayk CP, Van Grunsven PM, et al. Slowing the deterioration of asthma and COPD during bronchodilator therapy by adding inhaled corticosteroids: a four-year prospective study. Ann Intern Med 1993; 118:770778

6 Overbeek SE, Kerstjens HA, Bogaard JM, et al. Is delayed introduction of corticosteroids harmful in patients with obstructive airways disease (asthma and COPD)? The Dutch Chronic Nonspecific Lung Disease Study Group. Chest 1996; 110:35-41

7 Kerstjens HA, Brand PL, Hughes MD, et al. A comparison of bronchodilator therapy with or without inhaled corticosteroid therapy for obstructive airways disease: Dutch Chronic NonSpecific Lung Disease Study Group. N Engl J Med 1992; 327:1413-1419

8 Van Grunsven PM, Van Schayck CP, Molema J, et al. Effect of inhaled corticosteroids on bronchial responsiveness in patients with "corticosteroid naive" mild asthma: a meta analysis. Thorax 1999; 54:316-322

9 Pauwels R. Inhaled glucocorticosteroids and chronic obstructive pulmonary disease: how full is the glass? Am J Respir Crit Care Med 2002; 165:1579-1580

10 Fabbri LM, Hurd SS, the GOLD Scientific Committee. Global Strategy for the Diagnosis, Management and Prevention of COPD: 2003 update. Eur Respir J 2003; 22:1-2

11 Fletcher C, Peto R. The natural history of chronic airflow obstruction. BMJ 1977; 1:1645-1648

12 Burrows B, Bloom JW, Traver GA, et al. The course and prognosis of different forms of chronic airways obstruction in a sample of the general population. N Engl J Med 1987; 317:1309-1314

13 Peat JK, Woolcock AJ, Cullen K. Rate of decline of lung function in subjects with asthma. Eur J Respir Dis 1987; 70:171-179

14 Burge PS, Calverley PMA, Jones PW, et al. on behalf of the ISOLDE study investigators: randomised, double blind, placebo controlled study of fluticasone propionate in patients with moderate to severe chronic obstructive pulmonary disease; the ISOLDE trial. BMJ 2000; 320:1297-1303

15 Pauwels RA, Lofdahl CG, Laitinen LA, et al. Long-term treatment with inhaled budesonide in persons with mild chronic obstructive pulmonary disease who continue smoking: European Respiratory Society Study on Chronic Ob- 
structive Pulmonary Disease. N Engl J Med 1999; 340:19481953

16 Van den Boom G, Rutten-Van Mölken PMH, Molema J, et al. The cost-effectiveness of early treatment with fluticasone propionate 250 mug twice a day in subjects with obstructive airway disease. Am J Respir Crit Care Med 2001; 164:20572066

17 Sutherland ER, Allmers H, Ayas NT, et al. Inhaled corticosteroids reduce the progression of airflow limitation in chronic obstructive pulmonary disease: a meta-analysis. Tho$\operatorname{rax} 2003 ;$ 58:937-941

18 Spencer S, Calverley PM, Burge PS, et al. Health status deterioration in patients with chronic obstructive pulmonary disease. Am J Respir Crit Care Med 2001; 163:122-128

19 Paggiaro PL, Dahle R, Bakran I, et al. Multicentre randomised placebo-controlled trial of inhaled fluticasone propionate in patients with chronic obstructive pulmonary disease: International COPD Study Group. Lancet 1998; 351:773-780

20 National Institutes of Health. Highlights of the expert panel report 2: guidelines for the diagnosis and management of asthma. Bethesda, MD: National Institutes of Health, 1997; NIH publication 97-4051A

21 Lundbäck B, Gulsvik A, Albers M, et al. Epidemiological aspects and early detection of chronic obstructive airway diseases in the elderly. Eur Respir J 2003; 21(suppl 40):3s-9s

22 Van Grunsven PM, Van Schayck CP, Van Deuveren M. Compliance during long-term treatment with fluticasone propionate in subjects with early signs of asthma or chronic obstructive pulmonary disease (COPD): results of the Detection, Intervention, and Monitoring Program of COPD and Asthma (DIMCA) Study. J Asthma 2000; 37:225-234

23 American Thoracic Society. Standardization of spirometry, 1994 update. Am J Respir Crit Care Med 1995; 52:1107-1136

24 Dompeling E, Van Schayck CP, Folgering H, et al. Accuracy, precision and linearity of the portable flow-volume meter Microspiro HI-298. Eur Respir J 1991; 4:612-615

25 Quanjer PH, Tammeling GJ, Cotes JE, et al. Lung volumes and forced ventilatory flows: Report Working Party Standard- ization of Lung Function Tests, European Community for Steel and Coal; Official Statement of the European Respiratory Society. Eur Respir J 1993; 16(suppl):5-40

26 Cockcroft DW, Killian DN, Mellon JJ, et al. Bronchial reactivity to inhaled histamine: a method and clinical survey. Clin Allergy 1977; 7:235-243

27 Rutten-Van Molken MP, Custers F, Van Doorslaer EK, et al. Comparison of performance of four instruments in evaluating the effects of salmeterol on asthma quality of life. Eur Respir J 1995; 8:888-898

28 Torrance GW, Feeny DH, Furlong WJ, et al. Multiattribute utility function for a comprehensive health status classification system: Health Utilities Index Mark 2. Med Care 1996; 34:702-722

29 Guyatt GH, Berman LB, Townsend M, et al. A measure of quality of life for clinical trials in chronic lung disease. Thorax 1987; 42:773-778

30 Juniper EF, Guyatt GH, Willan A, et al. Determining a minimal important change in a disease-specific Quality of Life Questionnaire. J Clin Epidemiol 1994; 47:81-87

31 D. M. Hawkins. Point estimation of the parameters of piecewise regression models. Appl Stat 1976; 25:51-57

32 Van Grunsven PM, Van Schayck CP, Derenne JP, et al. Long-term effects of inhaled corticosteroids in chronic obstructive pulmonary disease: a meta analysis. Thorax 1999; 54:7-14

33 Vestbo J, Sorensen T, Lange $\mathrm{P}$, et al. Long-term effect of inhaled budesonide in mild and moderate chronic obstructive pulmonary disease: a randomised controlled trial. Lancet 1999; 353:1819-1823

34 Wise MD, Connet J, Weinmann G, et al. Effect of inhaled triamcinolone on the decline in pulmonary function in chronic obstructive pulmonary disease. N Engl J Med 2000; 343:1902-1909

35 Rijcken B, Schouten JP, Xu X, et al. Airway hyperresponsiveness to histamine associated with accelerated decline in $\mathrm{FEV}_{1}$. Am J Respir Crit Care Med 1995; 151:377-1382 